\title{
Robust Control Design for Uncertain Nonlinear Dynamic Systems
}

\author{
Sean P. Kenny, \\ Senior Research Engineer, NASA Langley Research Center, Hampton, VA, 23666 \\ Luis G. Crespo, \\ Senior Research Scientist, National Institute of Aerospace, 100 exploration way, Hampton, VA, 23666 \\ Lindsey Andrews, \\ Student, Mechanical Engineering Department, Old Dominion University, Norfolk, VA, 23508 \\ Daniel Giesy, \\ Research Mathematician, NASA Langley Research Center, Hampton, VA, 23666
}

\begin{abstract}
Robustness to parametric uncertainty is fundamental to successful control system design and as such it has been at the core of many design methods developed over the decades. Despite its prominence, most of the work on robust control design has focused on linear models and uncertainties that are non-probabilistic in nature. Recently, researchers have acknowledged this disparity and have been developing theory to address a broader class of uncertainties. This paper presents an experimental application of robust control design for a hybrid class of probabilistic and non-probabilistic parametric uncertainties. The experimental apparatus is based upon the classic inverted pendulum on a cart. The physical uncertainty is realized by a known additional lumped mass at an unknown location on the pendulum. This unknown location has the effect of substantially altering the nominal frequency and controllability of the nonlinear system, and in the limit has the capability to make the system neutrally stable and uncontrollable. Another uncertainty to be considered is a direct current motor parameter. The control design objective is to design a controller that satisfies stability, tracking error, control power, and transient behavior requirements for the largest range of parametric uncertainties. This paper presents an overview of the theory behind the robust control design methodology and the experimental results.
\end{abstract}

\section{Introduction}

This paper presents an application of a control design and analysis methodology for arbitrary dynamic systems in the presence of parametric uncertainty. The specific classes of uncertainty considered here include multidimensional sets and probability density functions (PDFs). In the context of this paper, multidimensional sets are simply regions in parameter space where the stability and performance of the dynamic system are to be assessed. A bounding set in two dimensions, for example, could be a rectangle that is defined by its center and semi-diagonal. Other bounding sets are permitted, e.g., circular, and elliptical, with no restrictions on the dimensionality of the uncertain parameter space. Bounding set models are typically used when little is known about the true variability of the uncertain parameters, or when the stability and performance of the system need to be certified over a specific range of uncertainties. The other form of uncertainty used in this work is the PDF that describes the relative likelihood for a random variable to occur at a given point. Uncertainty models based upon PDFs require more information to create, but yield likelihood information of stability and performance of the controlled system. Despite the fundamental differences in these two forms of uncertainty, a unified framework [1] has been developed to accommodate both simultaneously.

The primary objective of this paper is to apply the methods outlined in [1] to a hardware realization of the classic 


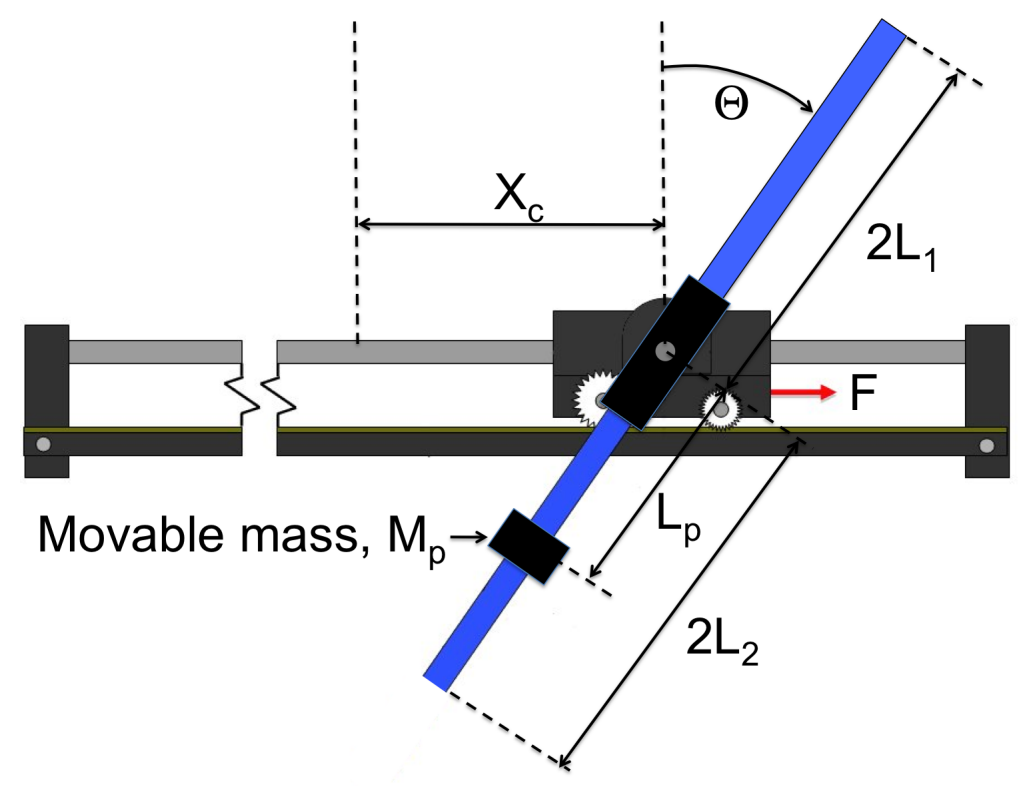

Fig. 1 Inverted pendulum with movable mass

inverted pendulum on a cart. This objective was broken into two tasks, one for pure analysis of both stability and performance, and one that focused on controller design for the nonlinear pendulum system. The analysis task was to assess the robustness of the system to parametric uncertainty in two parameters, whereas the design task was to synthesize an optimally robust fixed-architecture controller for the nonlinear system.

\section{Inverted Pendulum System}

Figure 1 illustrates the experimental apparatus used in this work. The system features a cart driven by a rack and pinion mechanism using a DC motor. The cart position is measured using an optical encoder coupled to the rack via an additional pinion. A pendulum mounted on the cart is free to rotate and its angle is measured via an optical encoder. The pendulum arrangement has been modified to accommodate the needs of this research. The primary modification is the addition of a rigid coupling connected to the pivot point to allow an additional link to be added. The modified system consists of two collinear links, the original upper link with length $2 L_{1}$, and a new lower link with length $2 L_{2}$. The lower link has been designed to permit the addition of a movable point mass, $M_{p}$, whose distance from the pivot point, $L_{p}$, is considered as one of the uncertain parameters. A total of two uncertain parameters were considered in this study and were distinct in that one was physically realizable by changing the location of a movable mass on the pendulum, and one was a simulation model parameter corresponding to the relationship of current input to torque output of the DC motor used to actuate the system. Both uncertain parameters have a profound effect on the stability, performance, and controllability of the dynamic system. For example, the movable mass degrades controllability of the system by requiring larger control signals to maintain stability and achieve adequate performance as the mass moves away from the pivot point.

\subsection{Model of the Dynamic System}

The classic inverted pendulum is well-studied and the equations of motion can be found in many references, e.g., [2]. However, the configuration used in this work departs slightly from the conventional inverted pendulum, so the equations of motion are stated here for completeness. 
The input force to the system is generated by applying a voltage to a DC motor to move the cart. The relationship between voltage, $V$, and applied force, $F$, can be represented as:

$$
\begin{aligned}
V & =I_{m} R_{m}+K_{m} K_{g} \omega_{g}=I_{m} R_{m}+K_{m} K_{g} \frac{\dot{X}_{c}}{r} \\
T & =K_{m} K_{g} I_{m} \\
F & =\frac{K_{m} K_{g} V}{R_{m} r}-\frac{K_{m}^{2} K_{g}^{2} \dot{X}_{c}}{R_{m} r^{2}}
\end{aligned}
$$

where $K_{m}$ is the DC motor torque constant, $K_{g}$ is the planetary gearbox gear ratio, $\omega_{g}$ is the angular velocity of the pinion gear, $R_{m}$ is the motor armature resistance, $I_{m}$ is the motor armature current, $r$ is the motor pinion diameter, and $V$ is the applied voltage. Using the relationships given in (1) together with the fundamental physics results in the following nonlinear equations of motion:

$$
\begin{gathered}
\ddot{X}_{c}=\frac{V N W-\dot{X}_{c} N W^{2}+\cos (\Theta) g \sin (\Theta) R_{m} P^{2}+\dot{\Theta}^{2} \sin (\Theta) R_{m} N P}{R_{m}\left(N M-\cos ^{2}(\Theta) P^{2}\right)} \\
\ddot{\Theta}=-\frac{P\left(g \sin (\Theta) R_{m} M+\cos (\Theta) \dot{\Theta}^{2} \sin (\Theta) R_{m} P+\cos (\Theta) V W-\cos (\Theta) \dot{X}_{c} W^{2}\right)}{R_{m}\left(N M-\cos ^{2}(\Theta) P^{2}\right)}
\end{gathered}
$$

where

$$
\begin{aligned}
W & =\frac{K_{g} K_{m}}{r} \\
P & =M_{2} L_{2}+M_{p} L_{p}-M_{l} L_{l} \\
N & =\frac{4}{3}\left(M_{2} L_{2}^{2}+M_{1} L_{1}^{2}\right)+M_{p} L_{p}^{2} \\
M & =M_{c}+M_{p}+M_{1}+M_{2}
\end{aligned}
$$

$X_{c}$ is the position of the cart and $\Theta$ is the angle of the pendulum. The angle $\Theta$ is measured counter-clockwise with zero defined when link one is pointing vertically up. Note that with this definition of $\Theta$, the angular displacement shown in figure 1 is actually negative. The remaining parameters above are defined as follows: $M_{c}$ is the mass of the cart, $M_{1}$ is the mass of link one, $M_{2}$ is the mass of link two, $L_{1}$ is the half-length of link one, $L_{2}$ is the half-length of link two, and $g$ is the gravitational acceleration. The MKS system of units are used throughout the paper.

Furthermore, it should be noted that equations given in (2) and (3) neglect damping.

\subsection{Linear System Model}

A general representation of the nonlinear equations given in (2) and (3) can be given as

$$
\dot{X}=\mathscr{F}(X, U, p)
$$

where $\mathscr{F}$ is a nonlinear function of the state vector $X$, the control input $U$, and the vector of uncertain parameters $p$. In this system, the vector of uncertain parameters is $p=\left[L_{p}, K_{m}\right]$.

For control design purposes, this nonlinear plant is linearized about the equilibrium point $(\bar{x}, \bar{u})$ satisfying $\mathscr{F}(\bar{x}, \bar{u}, p)=$ 0 . Deviations from the equilibrium values $\bar{x}(p)$ and $\bar{u}(p)$ are written as lowercase letters hereafter, e.g., $X=\bar{x}+x$ and $U=\bar{u}+u$. Linearization of (8) about this equilibrium point leads to the system

$$
\dot{x}=A(p) x+B(p) u+\eta(x, u, p)
$$


where

$$
A(p)=\left.\frac{\partial \mathscr{F}}{\partial X}\right|_{\bar{x}(p), \bar{u}(p)} \quad B(p)=\left.\frac{\partial \mathscr{F}}{\partial U}\right|_{\bar{x}(p), \bar{u}(p)}
$$

and $\eta(x, u, p)$ contains higher-order terms. Note that $A, B$ and the high-order terms depend on $p$. In a sufficiently small neighborhood of the equilibrium point the effect of the higher-order terms is negligible. The Linear Time Invariant (LTI) representation of the plant results from dropping the higher-order terms in Equation 96. This LTI system can be written as

$$
\begin{aligned}
& \dot{x}=A(p) x+B(p) u \\
& y=C x
\end{aligned}
$$

where $A(p)$ is the system matrix, $B(p)$ is the input matrix, $C$ is the output matrix, $x$ is the state vector, and $u$ is the input. This is applied to the inverted pendulum by choosing the state vector to be $x=\left[x_{c}, \theta, \dot{x_{c}}, \dot{\theta}\right]^{\top}$ and the control input to be the applied voltage; i.e., $u=V$. The equilibrium point about which we linearize is $\bar{x}(p)=[0,0,0,0]^{\top}$ and $\bar{u}(p)=0$. With these choices, the LTI representation matrices are:

$$
\begin{gathered}
A(p)=\left[\begin{array}{cccc}
0 & 0 & 1 & 0 \\
0 & 0 & 0 & 1 \\
0 & \frac{g P^{2}}{N M-P^{2}} & -\frac{N W^{2}}{R_{m}\left(N M-P^{2}\right)} & 0 \\
0 & -\frac{g P M}{N M-P^{2}} & \frac{P W^{2}}{R_{m}\left(N M-P^{2}\right)} & 0
\end{array}\right] \\
B(p)=\left[\begin{array}{c}
0 \\
0 \\
\frac{N W}{R_{m}\left(N M-P^{2}\right)} \\
-\frac{P W}{R_{m}\left(N M-P^{2}\right)}
\end{array}\right]
\end{gathered}
$$

and

$$
C=\left[\begin{array}{llll}
1 & 0 & 0 & 0 \\
0 & 1 & 0 & 0
\end{array}\right]
$$

\section{Control Architecture}

There have been numerous control architectures developed and successfully implemented on similar inverted pendulum systems. The objective of this work is not to introduce a new control architecture, but instead use one that is familiar to many with control design experience and then demonstrate methods to optimally robustify this fixed architecture controller to parametric uncertainties. The mathematical framework for robustness analysis and control tuning, covered in Section 4, is capable of handling a wide array of control architectures and plant models, linear or nonlinear, with arbitrary parameter dependence. The primary objective of the control system is for the cart to track a periodic reference signal while maintaining stability and adequate performance. One period of the commanded reference signal is given in figure 2. See Section 4.3 for a detailed description of performance requirements. 


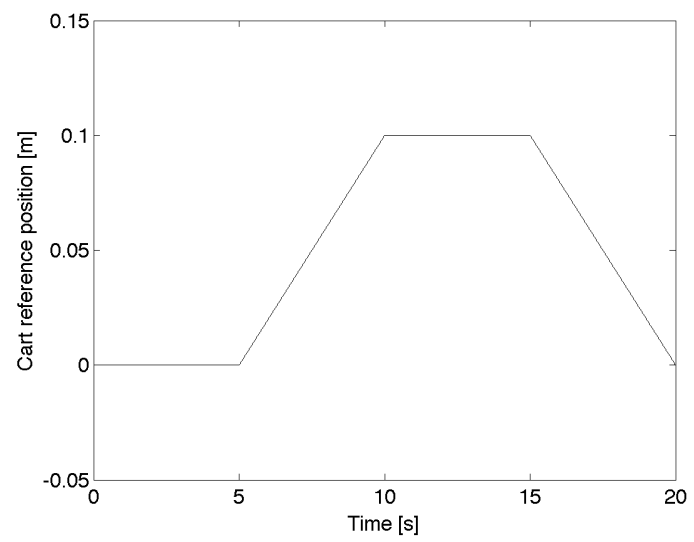

Fig. 2 Cart position reference signal

\subsection{Linear Quadratic Regulator Control with State Estimation}

A Linear Quadratic Regulator (LQR) control together with state estimation constitute the control architecture. The estimator was necessary since only the two positional states (cart position and pendulum angle) of the four states were measured outputs. Conventional steady-state implementations of LQR control works by multiplying the states by a constant gain matrix, $\mathrm{K}$, to produce the plant control signal. The gain matrix is generated by minimizing the following cost function:

$$
J=\int_{0}^{\infty}\left[x^{\top} Q x+u^{\top} R u\right] d t
$$

In this cost function, weighting matrices $Q \geq 0$ and $R>0$ are used to penalize deviation of the states from zero and penalize control energy. Choosing these weighting matrices is the fundamental task of the control designer. In the results to follow, these weighting matrices were chosen using a numerical optimization strategy.

Two additional features were added to the control architecture to achieve better tracking and steady state error performance. The first was the addition of a feed-forward control term using the known reference command, and the second was an integral error feedback term on cart position error. The feed-forward command was added to regulate the system about the reference command instead of regulating it about $x(t)=0$. This resulted in the following control signal:

$$
u(t)=-K\left(x-x_{r e f}\right)=-K x+K x_{r e f}
$$

where $x_{r e f}$ is the 4-element state reference vector. The second feature is an integral error feedback, which is added by augmenting the nominal state vector with an additional element. This additional element is an error between the cart's actual position and its commanded position. The total control signal is:

$$
u(t)=-K\left(x-x_{r e f}\right)-K_{i} e
$$

where $e$ is defined as:

$$
e=\int_{0}^{t}\left(x_{c_{r e f}}(\tau)-x_{c}(\tau)\right) d \tau
$$

with $x_{c_{r e f}}$ defined as: $x_{c_{r e f}}=[1,0,0,0] x_{r e f}$. Note that integral action is only added to the cart error signal and not to the full measurement vector. The scalar gain term, $K_{i}$, is the last element in the gain vector obtained by solving an LQR problem on an augmented system that includes the error state. 


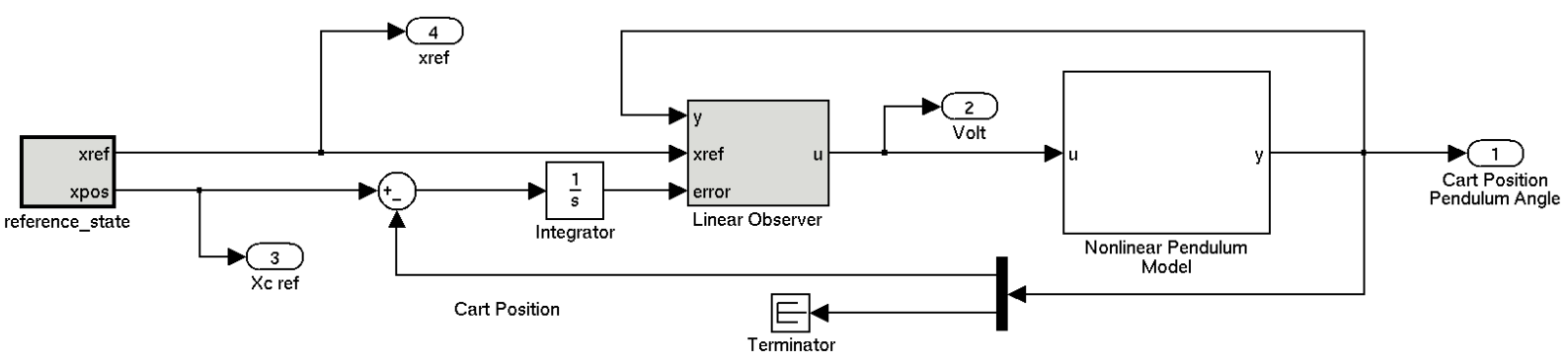

Fig. 3 Simulink Model of the Control Architecture

The remaining element of the control system is the state estimator which is a standard implementation of the Kalman filter. As is standard, the state estimate, $\hat{x}$, is used to replace $x$ in (17) for implementation on the inverted pendulum hardware. In the design and analysis that follows, the state estimator parameters are fixed. This is consistent with current practices, since the true values of the parameters are not known prior to implementation. The Kalman filter gains in this work were computed according to the system performance when the uncertainties assume their nominal values.

A Simulink ${ }^{\circledR}$ implementation of the control architecture is shown in figure 3 . A linear representation of this system is as follows:

$$
\left[\begin{array}{c}
\dot{x} \\
\dot{x} \\
\dot{e}
\end{array}\right]=\left[\begin{array}{ccc}
A-L C-B K & L C & -B K_{i} \\
-B K & A & -B K_{i} \\
0 & -C_{1} & 0
\end{array}\right]\left[\begin{array}{l}
\hat{x} \\
x \\
e
\end{array}\right]+\left[\begin{array}{c}
B K \\
B K \\
C_{1}
\end{array}\right] x_{r e f}
$$

where $L$ is the Kalman gain matrix, and $C_{1}=[1,0,0,0]$.

\section{Mathematical Framework for Robustness Analysis and Control Tuning}

\subsection{Robustness Analysis}

A brief introduction to the methodology proposed in [1] is presented next. Consider the closed-loop dynamic system

$$
\dot{X}=\mathscr{F}(X, U, p, r)
$$

where $r$ is a generic representation of a reference signal, with $x_{\text {ref }}$ in Equation 19 as a specific realization of the reference signal.

The control input can be represented as

$$
U=U(X, r, K)
$$

where the function $U$ refers to a particular control structure and $K \in \mathbb{R}^{\operatorname{dim}(K)}$ is the set of free parameters, to be chosen by the control engineer (e.g., control gains), that fully prescribe the controller. The controller is deemed acceptable if the closed-loop response satisfies a set of requirements. These requirements, which are represented by a set of inequality constraints on selected output metrics, depend on the uncertain parameter vector $p$. The control system is deemed acceptable for a given realization, $p$, of the uncertain parameters if all inequalities are satisfied. Specifically, the satisfaction of the vector inequality

$$
g(p, r, K)<0
$$


implies the satisfaction of all the requirement 1 , and where $g \in \mathbb{R}^{\operatorname{dim}(g)}$. These constraints partition the uncertain parameter space into two sets, the failure domain $F=\left\{p: g_{i}(p) \geq 0\right.$, for some $\left.i\right\}$, where at least one requirement is violated, and the safe domain $S=\{p: g(p)<0\}$, where all requirements are satisfied. The term "satisfactory performance", which is to be captured in $g$, is broad in scope and refers to acceptable ranges of variation in metrics of interest. These metrics may correspond to specific performance specifications as well as to basic notions of goodness. For instance, if $y(p, t)$ is the system output to a step-input and $t_{a s}$ is the admissible settling time, the corresponding requirement function is $g_{\text {settling }}=t_{s}-t_{a s}$ where $t_{s}$ is the minimal time for which $|y(p, t)-y(p, \infty)| \leq 0.99$ for all $t>t_{s}$.

The parameter realization $\bar{p} \in \mathbb{R}^{\operatorname{dim}(p)}$, called the nominal operating conditions point, denotes our best estimate of the actual value of $p$. The objective of this robustness analysis is to determine the largest deviation of $p$ from $\bar{p}$ for which all the requirements are met. In this paper, such a deviation is prescribed as a hyper-rectangular set of fixed proportions. Note that the hyper-rectangle centered at $\bar{p}$ with semi-diagonal vector $m>0$ is given by

$$
R(\bar{p}, m)=\{p: \bar{p}-m \leq p \leq \bar{p}+m\}
$$

The notions required to calculate this deviation are introduced next.

A homothetic deformation results from a uniform, radial expansion or contraction of the space about a fixed point. The distance from any point in the space to the fixed point changes by a factor $\alpha$ after the deformation. This factor is called the similitude ratio of the homothetic deformation. Note that if $\alpha$ is greater than 1 , the deformation is an expansion, while if $\alpha$ is less than 1, the deformation is a contraction. A reference set, denoted as $\Omega \subset \mathbb{R}^{\operatorname{dim}(p)}$, is deformed with respect to its geometric center $\bar{p}$. Intuitively, one can imagine that $\Omega$ is being deformed with respect to $\bar{p}$ until its boundary just touches the failure domain boundary $\partial F$. This deformation is called the maximal deformation. The set resulting from this deformation, denoted as $M \subseteq \mathbb{R}^{\operatorname{dim}(p)}$, is the maximal set. A critical parameter value $(\mathrm{CPV})$, denoted as $\tilde{p} \in \mathbb{R}^{\operatorname{dim}(p)}$, is (one of) the point(s) where the maximal set touches $\partial F$. This point is a worst-case uncertainty combination associated with $\Omega$. The critical similitude ratio (CSR), denoted by $\tilde{\alpha} \in \mathbb{R}$, is the similitude ratio of that deformation and is a non-dimensional metric proportional to the separation between the point $\bar{p}$ and the failure domain. The parametric safety margin (PSM), denoted as $\rho \in \mathbb{R}$ is its dimensional equivalent. The values taken on by the CSR and the magnitude of the PSM are proportional to the size of the maximal set and measure the separation between $\bar{p}$ and $\partial F$. The PSM is assigned a negative value if the controller does not even satisfy the requirements at $\bar{p}$. If the PSM is zero, the controller exhibits no robustness because there is an arbitrarily small deviation from $\bar{p}$ leading to a requirement violation. If the PSM is positive, the requirements are satisfied at $\bar{p}$ and in its vicinity. The larger the PSM, the larger the $\Omega$-shaped vicinity.

Figure4 4 illustrates some of these variables when two requirements are present. The CPV, $\tilde{p}$, the nominal parameter point, $\bar{p}$, the reference set (region within dashed line) and the maximal set corresponding to deformations in the safe (blue) and failure (red) domains are shown. Note that by construction, the maximal set is fully contained in the safe or failure domains.

The formulation enabling sizing the maximal deformation is as follows. If $\Omega=R(\bar{p}, m)$, the CPV, CSR, PSM and maximal sets corresponding to the $i$ th requirement are

$$
\begin{gathered}
\tilde{p}^{i}=\underset{p}{\operatorname{argmin}}\left\{\|p-\bar{p}\|_{m}^{\infty}: \sigma_{i} g_{i}(p, r, K) \geq 0, p_{\min } \leq p \leq p_{\max }\right\} \\
\tilde{\alpha}_{i}=\frac{\left\|\tilde{p}^{i}-\bar{p}\right\|_{m}^{\infty}}{\|m\|} \\
\rho_{i}=\sigma_{i} \tilde{\alpha}_{i}\|m\| \\
M^{i}=R\left(\bar{p}, \tilde{\alpha}_{i} m\right)
\end{gathered}
$$

where $\|x\|_{m}^{\infty}=\max _{i}\left\{\left|x_{i}\right| / m_{i}\right\}, p_{\min }$ and $p_{\max }$ bound the region of interest, and $\sigma_{i}=1$ if $g_{i}(\bar{p})<0$, otherwise $\sigma_{i}=-1$. Note that $\sigma_{i}$ can reverse the inequality sign of the constraint. This is needed since deformations centered at a nominal point in the safe domain require a different formulation than those where the nominal point is in the failure domain (See figure 47. While in the former case all points within the maximal set must satisfy all constraints, in the latter case

\footnotetext{
${ }^{1}$ For the reminder of the paper is assumed that vector inequalities hold component-wise, vector and set super-indices denote a particular realization, and vector sub-indices denote a particular component, e.g., $p_{i}^{j}$ is the $i$ th component of $p^{j} \in \mathbb{R}^{\operatorname{dim}(p)}$.
} 


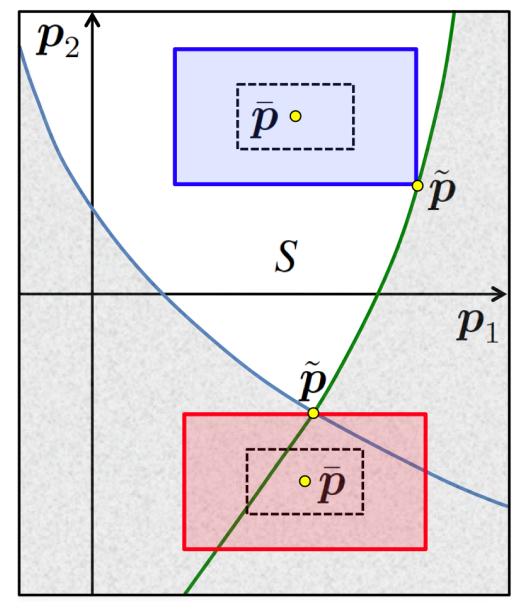

Fig. 4 Maximal deformations in the safe and failure domains

all points must violate at least one constraint. The value of the controller's parameters $K$ and the commands in $r$ are set a priori and kept fixed during the analysis. While $K$ can be prescribed according to any control design practice, $r$ is prescribed to achieve the ultimate goal of the system.

The CPV, CSR, PSM and maximal sets associated with all requirements are given by Equations (24 27) after replacing $g_{i}$ with $\max _{i}\left(g_{i}\right)$. The resulting values are qualified as aggregate since they take all requirements into account. If $\bar{p} \in F$, these aggregate values coincide with those corresponding to the individual requirement attaining the smallest PSM, e.g., $\tilde{\alpha}=\tilde{\alpha}_{j}$ where $j=\operatorname{argmin}\left(\rho_{i}\right)$. Therefore, for a maximal safe set, the aggregate PSM is the worst-case individual PSM.

The calculation of the maximal deformation requires solving an standard optimization problem. In this work, a gradient-based nonlinear constrained optimization was used. This problem class is, in general, non-convex when the dependency of the vector $g_{i}$ of requirement functions on the uncertainty $p$ is nonlinear. In any non-convex optimization problem there is always the possibility of convergence to a non-global optimum. When this occurs, the CPV resulting from the numerical optimization is mistaken and the corresponding maximal set intrudes into the failure region. This intrusion yields to a spuriously larger PSM. Absolute guarantees are not possible, but a variety of algorithmic safeguards can be used to deal with this deficiency. For instance, $g$ can be evaluated at a few sample points in the maximal set and if one happens to fall into the failure domain, it can be used as an initial condition in a subsequent optimization. Requirements functions with a polynomial parameter dependency can be handled using the methods in [3]. These methods, which are based on linear matrix inequalities and Bernstein expansions, do not suffer from this potential drawback and guarantee convergence to actual maximal deformation. Notice however, that in most practical applications the explicit functional form of $g$ is unknown. This intrinsic feature of the problem precludes the usage of convexity conditions from the outset.

\subsection{Control Tuning}

Ultimately, a good controller should satisfy the stability and performance requirements imposed upon the closed-loop system with sufficiently large robustness margins. The PSM and CSR introduced above are examples of such margins. A computational framework, also used in [4], enables searching for the set of controller gains that maximize these margins. This framework is introduced next.

Equation (22) indicates that the geometry of the failure domain, thus the CSR and the PSM, is a function of the control parameter $K$. Recall that this parameter is kept fixed when a robustness analysis is performed. A control design challenge is to find the value of $K$ for which the closed-loop system exhibits satisfactory robustness characteristics. Specifically, an optimal design will be one that has the largest overall PSM for the given control structure. This entails maximizing the separation between the nominal operating condition point $\bar{p}$ and the failure domain $F$, 
so the largest maximal set is attained. Since the resulting controllers tolerate the largest deviations from nominal operating conditions before violating any requirement, they are called optimally robust. In the presence of conflicting design objectives, an optimally robust controller trades off the PSMs of all individual requirements to attain the largest aggregate PSM.

Two optimization-based formulations for pursuing the optimally robust design point $\tilde{K}$ are presented next. The first one evaluates robustness via the exact CSR. The second one uses an approximation to the CSR rooted in a multi-point approximation to the maximal set. The approximate nature of this formulation may not lead to controllers with the intended features. However, its relaxed computational demands may justify the potential drawbacks associated with it.

\subsubsection{Enlarging the Maximal Set}

A formulation based on the exact overall CSR is given by

$$
\tilde{K}=\underset{K}{\operatorname{argmax}}\{\tilde{\alpha}(K): h(\bar{p}, K)>0\}
$$

where the vector constraint $h(K, \bar{p})>0$ is used to enforce additional characteristics to the controller based on standard control metrics. For instance, $h=P M(K, \bar{p})-60^{\circ}>0$ ensures that the feedback loop corresponding to the plant under nominal operating conditions has a phase margin of more than 60 degrees. Examples of other performance/stability metrics are time delay, settling time, disturbance rejection, noise attenuation, reference tracking, and control energy. These metrics can be extracted from the time and/or frequency domains and may correspond to linear and nonlinear representations of the plant dynamics.

Recall that calculating the CSR $\tilde{\alpha}$ entails solving an optimization problem. Therefore, this formulation has an optimization in the outer loop and another one in the inner loop. While the outer loop searches for the robustly optimal gains $\tilde{K}$, the inner one searches for the CSR corresponding to the design point under consideration. Nested optimizations, such as Equation (28) and those resulting from worst-case-based design policies, impose stringent computational demands. Such demands can be substantially mitigated by using multi-point approximations.

\subsubsection{Enlarging an Approximation to the Maximal Set}

The variables $\hat{K}$ and $\hat{\alpha}$, which are estimates of $\tilde{K}$ and $\tilde{\alpha}(\tilde{K})$ respectively, are given by

$$
\langle\hat{K}, \hat{\alpha}\rangle=\underset{K, \alpha}{\operatorname{argmax}}\left\{\alpha: \max _{i \leq \operatorname{dim}(g), j \leq n}\left\{g_{i}\left(\bar{p}+\alpha\left(p^{j}-\bar{p}\right), r, K\right)\right\}<0, \alpha \geq 0, h(\bar{p}, K)>0\right\},
$$

where $\left\{p^{1}, p^{2}, \ldots, p^{n}\right\}$ are parameter-points on the surface of $R(\bar{p}, m)$. These points should be uniformly distributed over the surface. Note that this formulation replaces the inner optimization loop in Equation (28) with a multi-point constraint over parameter points lying on the surface of a maximal set estimate. Equation (29) may lead to suboptimal designs, for which $\hat{\alpha}>\tilde{\alpha}(\tilde{K})$ and $M(\hat{K}) \subset M(\tilde{K})$, because the satisfaction of the multi-point constraint does not guarantee the containment of the maximal set estimate by the safe domain. The formulation of Section 4.1 can be used to determine if this anomaly has occurred.

Since the robustness analysis and tuning procedures above identify the critical combination of uncertainties and/or failures (i.e., the CPV) the particular control architecture is more sensitive to, we expect the resulting controllers to be safer than those validated by brute force Monte Carlo simulations.

\subsection{Application to the Inverted Pendulum}

The components of the uncertain parameter $p$ are the distance, $L_{p}$, of the mass $m_{p}$ to the pivot point, and the motor torque constant $K_{m}$. These parameters are free to assume any non-negative value, i.e., $p_{\min }=[0,0]^{\top}, p_{\max }=$ $[0.330 \mathrm{~m}, \infty]^{\top}$. The nominal operating condition point is $\bar{p}=[0.165 \mathrm{~m}, 0.00767 \mathrm{~N}-\mathrm{m} / \mathrm{Amp}]^{\top}$. The robustness of the controller to uncertainty in the value of these two parameters is evaluated and improved using the methods above. The reference command is given by $r=\left[x_{\text {ref }}, \dot{x}_{\text {ref }}, 0,0\right]^{\top}$, where $x_{\text {ref }}$ is the periodic signal shown in Figure 2 The design 
parameters $K$ are diagonal elements of the $Q$ and $R$ matrices of the LQR formulation of Section 3 Denote by $K_{\text {base }}$ the gains of a baseline controller attaining satisfactory performance at $p=\bar{p}$, and $t_{f}$ the final simulation time. The closed-loop requirements defining the constraint function $g$ are as follows:

1) Command tracking: the requirement $g_{1}<0$, where

$$
g_{1}=\int_{0}^{t_{f}}\left(x_{c_{r e f}}(t)-x_{c}(p, r, K, t)\right)^{2} d t-\gamma_{1} \int_{0}^{t_{f}}\left(x_{c_{r e f}}(t)-x_{c}\left(\bar{p}, r, K_{\text {base }}, t\right)\right)^{2} d t
$$

and $\gamma_{1}>1$ ensures satisfactory tracking of the reference command.

2) Actuation: the requirement $g_{2}<0$, where

$$
g_{2}=\int_{0}^{t_{f}} u^{2}(p, r, K, t)-\gamma_{2} u^{2}\left(\bar{p}, r, K_{\mathrm{base}}, t\right) d t
$$

and $\gamma_{2}>1$ avoids over actuation.

3) Peak overshoot: the requirement $g_{3}<0$, where

$$
g_{3}=\max _{t}\left\{x_{c}(p, r, K, t): t_{0} \leq t \leq t_{f}\right\}-\gamma_{3} \max _{t}\left\{x_{c}\left(\bar{p}, r, K_{\text {base }}, t\right): t_{0} \leq t \leq t_{f}\right\}
$$

and $\gamma_{3}>1$ bounds the peak overshoot of the response.

4) Settling time: the requirement $g_{4}<0$, where

$$
\begin{aligned}
g_{4} & =t_{s}(p, r, K)-\gamma_{4} t_{s}\left(\bar{p}, r, K_{\mathrm{base}}\right) \\
t_{s}(p, r, K) & =\min _{t}\left\{\left|x_{c}(p, r, K, t)-0.1\right|<\varepsilon \text { for all } t \text { satisfying } t_{s}<t<5 \mathrm{~s}\right\}
\end{aligned}
$$

and $\gamma_{4}>1$ imposes an upper bound to the settling time.

5) Local Stability: the requirement $g_{5}<0$, where

$$
g_{5}=\max \{\operatorname{Real}\{\text { Eigenvalues }\{A(p, K)\}\}\}
$$

and $A$ is the closed-loop system matrix of the LTI system, ensures that the up-right position of the pendulum is locally stable.

The constraint functions corresponding to the first two requirements are evaluated using the $L_{2}$ norm, and therefore, they are a function of the transient response. Notice that the dependency of $g$ on $p$ assumes an unknown and implicit functional form. Also notice that evaluating $g$ for a particular realization of $p$ requires simulating the closed-loop response and calculating the five constraint functions comprising $g$. Further notice that while the notions of goodness supporting the requirement functions are fairly universal, their particular functional form is not.

\section{Results}

\subsection{Baseline Controller}

This controller, to be denoted as $K_{\text {base }}$, was designed using the structure presented in Equation 17 based on the closedloop performance at $p=\bar{p}$. The parameters determining $K$ are the $\mathrm{LQR}$ matrices $Q=\operatorname{diag}\left\{[40,0,0,0,50]^{\top}\right\}$ and $R=10^{-3}$. This controller performed well in both the simulation and experimental settings. Figure 5 shows the number of requirement violations in the parameter space and the maximal set when the time responses, thus the constraint function $g$, are evaluated using a linear simulation. Note that the safe domain, where all requirements are satisfied, corresponds to the green region. The color scale indicates the number of constraints violated, with black corresponding to all five constraints violated. The colored lines correspond to constraint boundaries for each of the 

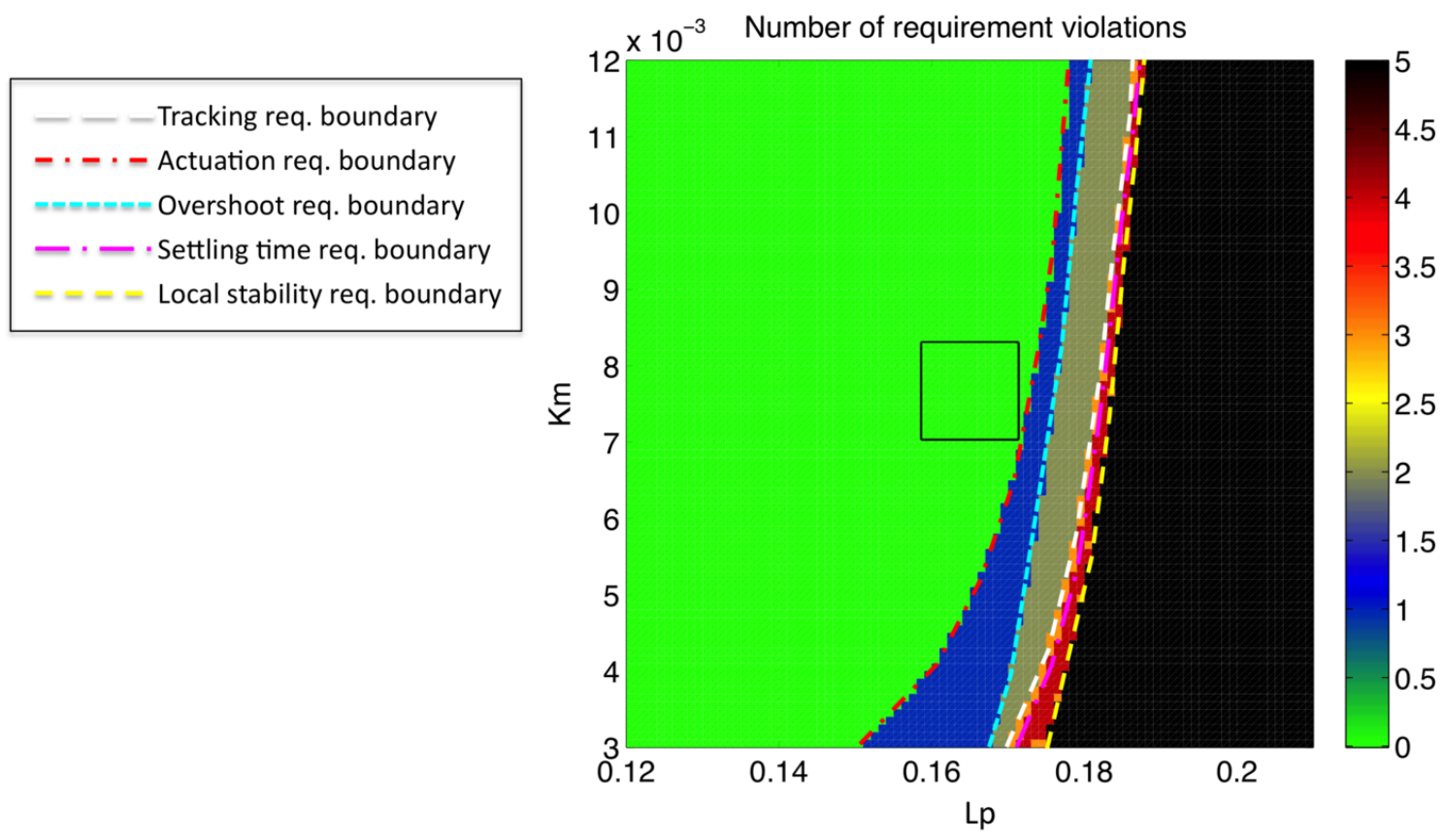

Fig. 5 Number of requirement violations in the parameter space and maximal set for $K_{\text {base }}$ using linear simulation

five constraints. The corresponding PSM is $\rho=6.4 \times 10^{-3}$. This figure, as some that follow, was generated by simulating the closed-loop response at the points of an uniformly spaced grid, calculating the constraint function $g$ at each point, and counting the number of non-negative components of each $g$. This large number of simulations (over 8000), which are not required to calculate the maximal deformation, has only been made for illustrative purposes.

Figure 6 shows the number of requirement violations and the maximal set for $K_{\text {base }}$ when evaluated using the nonlinear simulation. The corresponding PSM is $\rho=1.6 \times 10^{-2}$. This larger PSM obtained from the nonlinear evaluation model is consistent with the design practice used for determining $K_{\text {base. }}$. The process was an iterative technique of modifying $Q$ and $R$ in the laboratory and evaluating the closed-loop performance on hardware.

By definition, the CPV is a point on the surface of the maximal set that touches the boundary of the failure domain. Some of the figures may misleadingly show that the CPV is at the interior of the safe domain. This is a mere consequence of the manner in which the figures are generated: they show a piecewise constant function over a uniform partition of the parameter space. The same value/color is assigned to all parameter realizations constituting an element of the partition. That value corresponds to the center of the box. The actual function, which is used when calculating the maximal deformation, may vary across any given uncertain parameter range.

\subsection{Tuned Controller 1: Designed based on Linear Simulation}

The application of the control tuning procedure above using time responses of the linearized model yields controller $K_{\text {tuned-1. }}$. The design parameters used throughout this study are the $Q$ and $R$ parameters of the LQR controller. The controller parameters for this linearized system model are $Q=\operatorname{diag}\left\{\left[7.67,0.28,7.62,1.4 \times 10^{-3}, 79.94\right]^{\top}\right\}$ and $R=$ $3.2 \times 10^{-3}$. Figure 7 shows the corresponding uncertain parameter space. The corresponding PSM, which is equal to $\rho=3.965 \times 10^{-2}$, is more than 6 times larger than that of the baseline. This illustrates the effectiveness of the control tuning procedure. Figure 8 shows the linear and nonlinear time responses of $K_{\text {tuned-1 }}$ for $p=\left[0.2,4 \times 10^{-3}\right]^{\top}$. Note that the more oscillatory nature of the nonlinear response makes the controller unacceptable.

A robustness analysis of $K_{\text {tuned-1 }}$ based on the nonlinear response leads to $\rho=6 \times 10^{-3}$. This PSM is 6.5 times smaller than that of the linear response. Figure 9 shows the corresponding uncertain parameter space. Major differences between the linear analysis of figure 7 and the nonlinear analysis of figure 9 are apparent. The requirements 


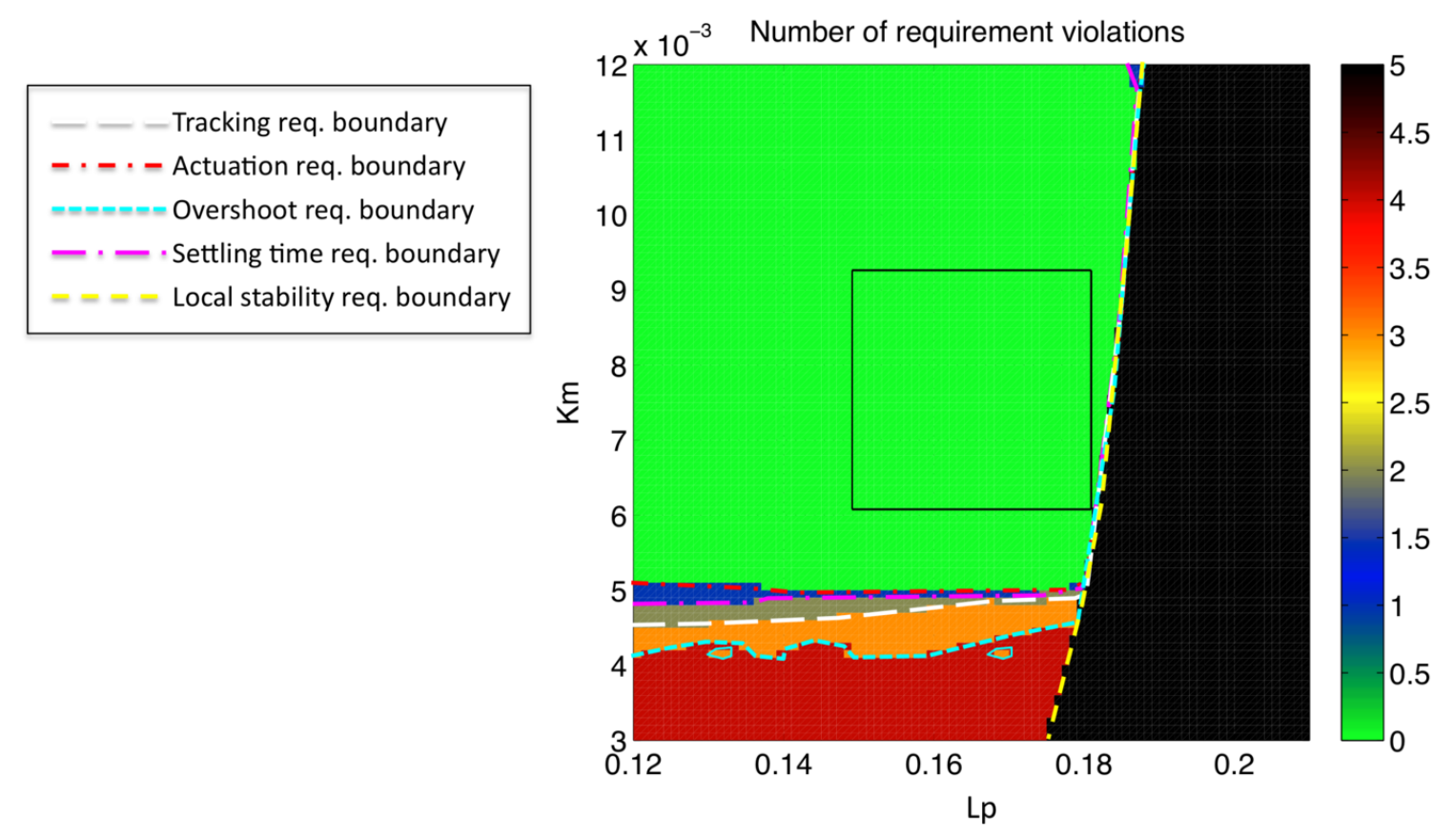

Fig. 6 Number of requirement violations in the parameter space and maximal set for $K_{\text {base }}$ using nonlinear simulation
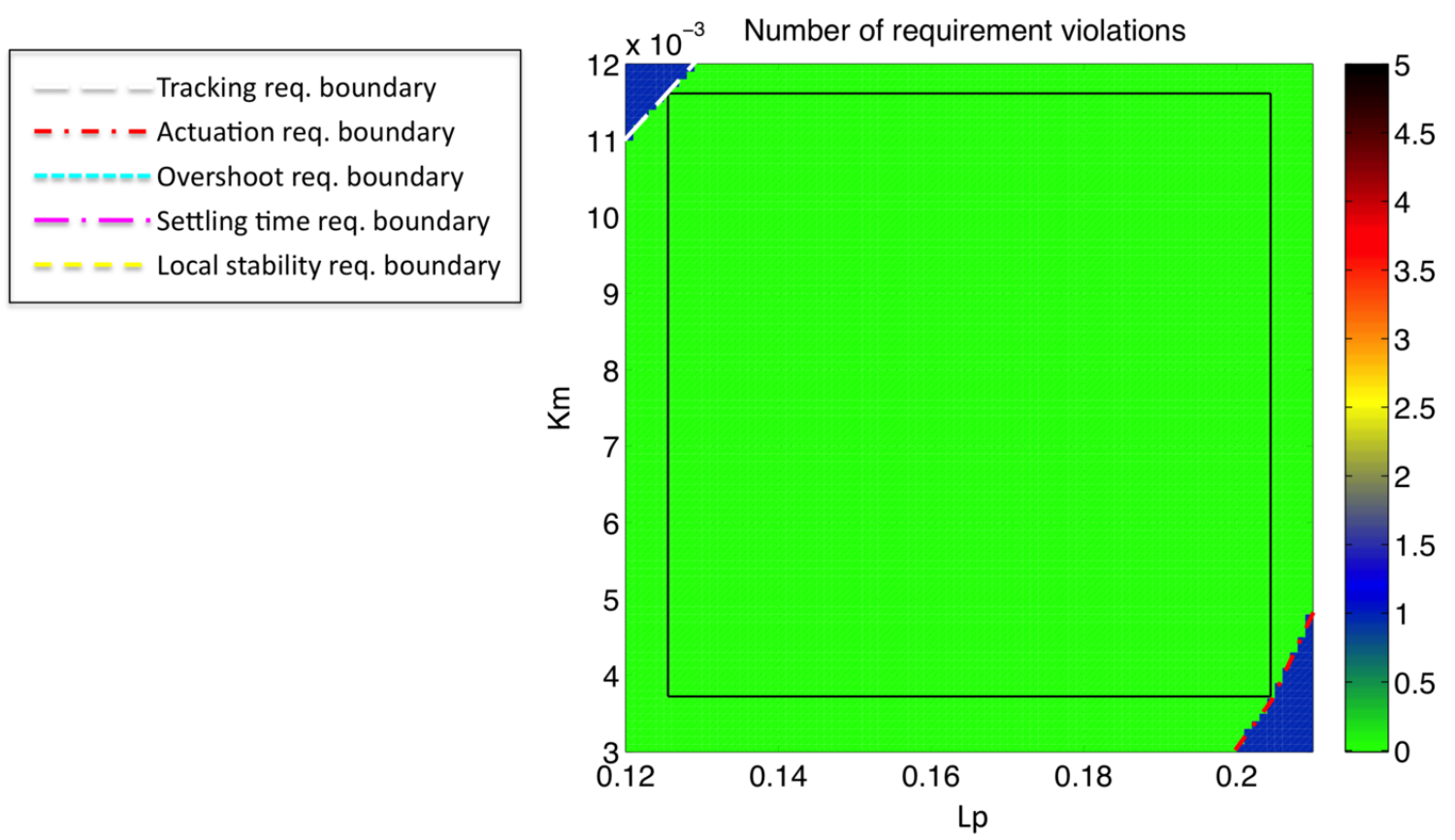

Fig. 7 Number of requirement violations in the parameter space for $K_{\text {tuned-1 }}$ according to the linear simulation 

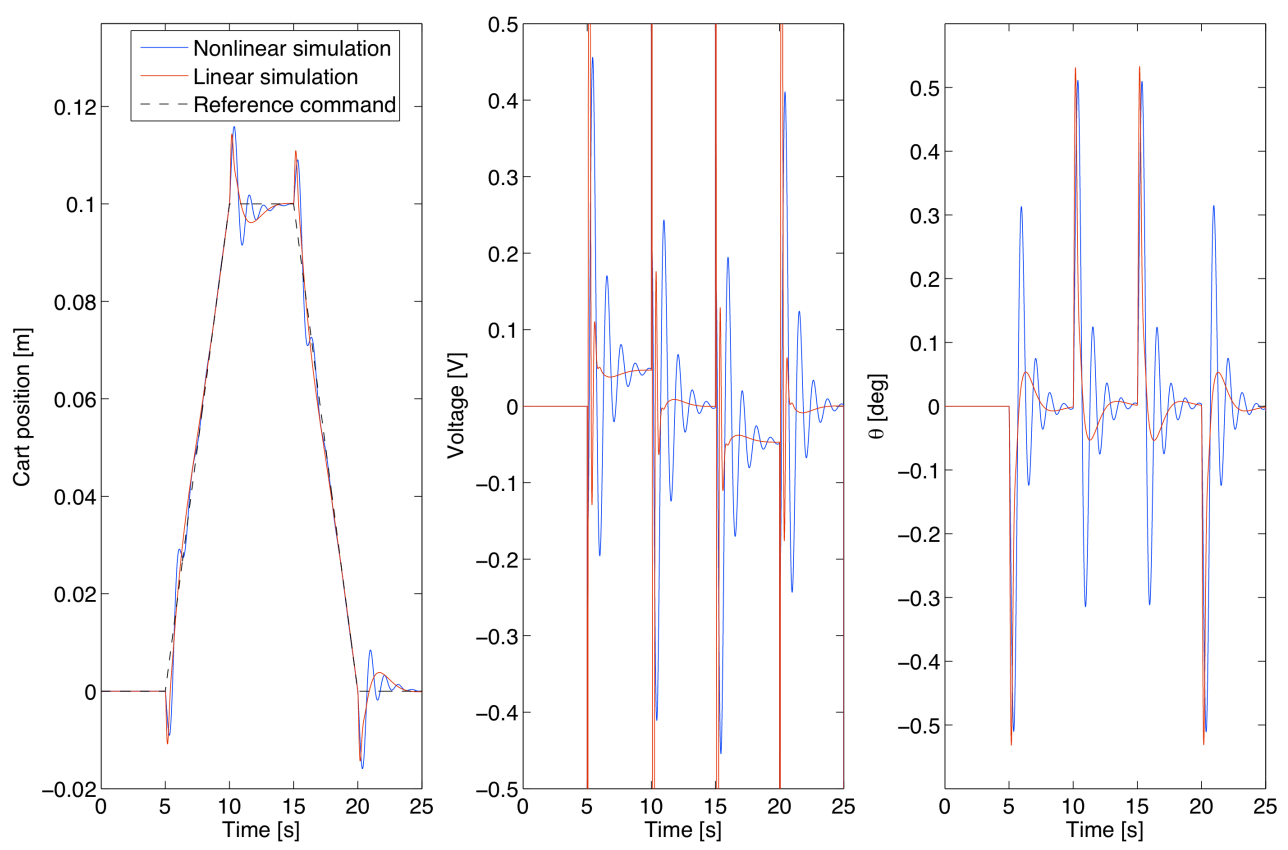

Fig. 8 Time responses for controller $K_{\text {tuned-1 }}$ at $p=\left[0.2,4 \times 10^{-3}\right]$

based on the $L_{2}$ norms, whose satisfaction depend upon the transient response, are the most affected.

\subsection{Tuned Controller 2: Designed based on Nonlinear Simulation}

The application of the control tuning procedure above using time responses of the nonlinear model equations, as given in 22 and 3 , yields controller $K_{\text {tuned-2. }}$. This controller parameters are $Q=\operatorname{diag}\left\{\left[8.20,10.63,0.19,10^{-4}, 42.46\right]^{\top}\right\}$ and $R=1.04 \times 10^{-4}$. Figure 10 shows the corresponding uncertain parameter space. The corresponding PSM is equal to $\rho=2.34 \times 10^{-2}$. As expected, this controller is more robust than $K_{\text {tuned-1 }}$ according to the nonlinear response. Note the transition between the safe domain (green area) and the region where the system is unstable (black area) may occur without a progressive and noticeable degradation in the transient response, i.e., the violation of $g_{5}<0$ resulting from a small drift in $p$ may not be preceded by any other requirement violation.

The robustness analysis of $K_{\text {tuned-2 }}$ according to the linear response leads to $\rho=3.7 \times 10^{-3}$. Figure 11 shows the corresponding number of requirement violations in uncertain parameter space. As before, significant differences between the linear and nonlinear analyses are present. These differences, which are the result of model-form uncertainty, highlight the need for assessing the dependence of the high order terms in Equation (9) on the uncertain parameters.

Experimental results for tuned controller 2 are shown in figures 12 and 13 . Figure 12 shows two cycles of the cart tracking response for the uncertain parameter point $p=\left[0.165,7.67 \times 10^{-3}\right]$. This point is well within the maximal set (see figure 10] and therefore we should expect a reasonable system response. Qualitatively the system response is good, however, due to additional unmodeled effects, such as: cart and pendulum damping, drive pinion eccentricity, drive rack flexibility, data and power cable frictional effects, etc., the true system response slightly differs from the simulated response. Figure 13 shows the cart tracking response for the uncertain parameter point $p=\left[0.20,7.67 \times 10^{-3}\right]$. This point is outside of the maximal set (where all five requirements fail) and therefore we should expect an unacceptable system response. Indeed this is the case as the system tracking response is unacceptably poor. It should be noted that if the system was perfectly linear then the response would be unstable yielding unbounded output. Instead a limit cycle oscillation is observed, which is consistent with the nonlinear system. 
_ - Tracking req. boundary

- - - - Actuation req. boundary

$=m=m=-$ Overshoot req. boundary

- - - Settling time req. boundary

$=--=$ Local stability req. boundary

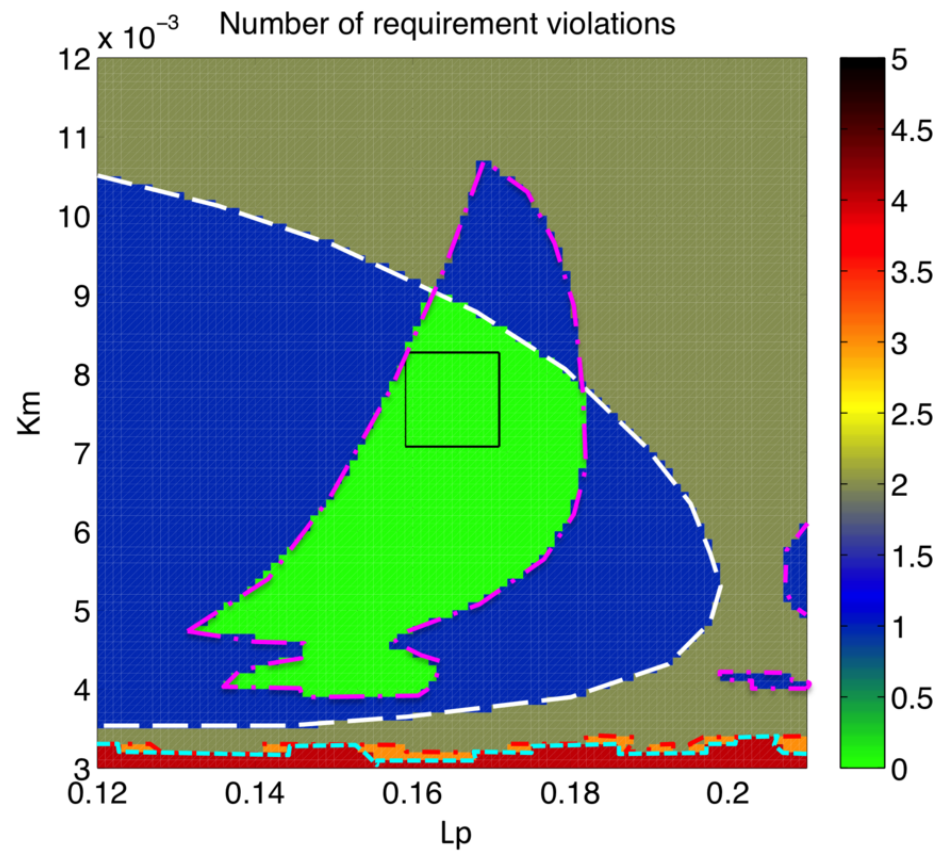

Fig. 9 Number of requirement violations in the parameter space and maximal set for $K_{\text {tuned-1 }}$ according to the nonlinear simulation
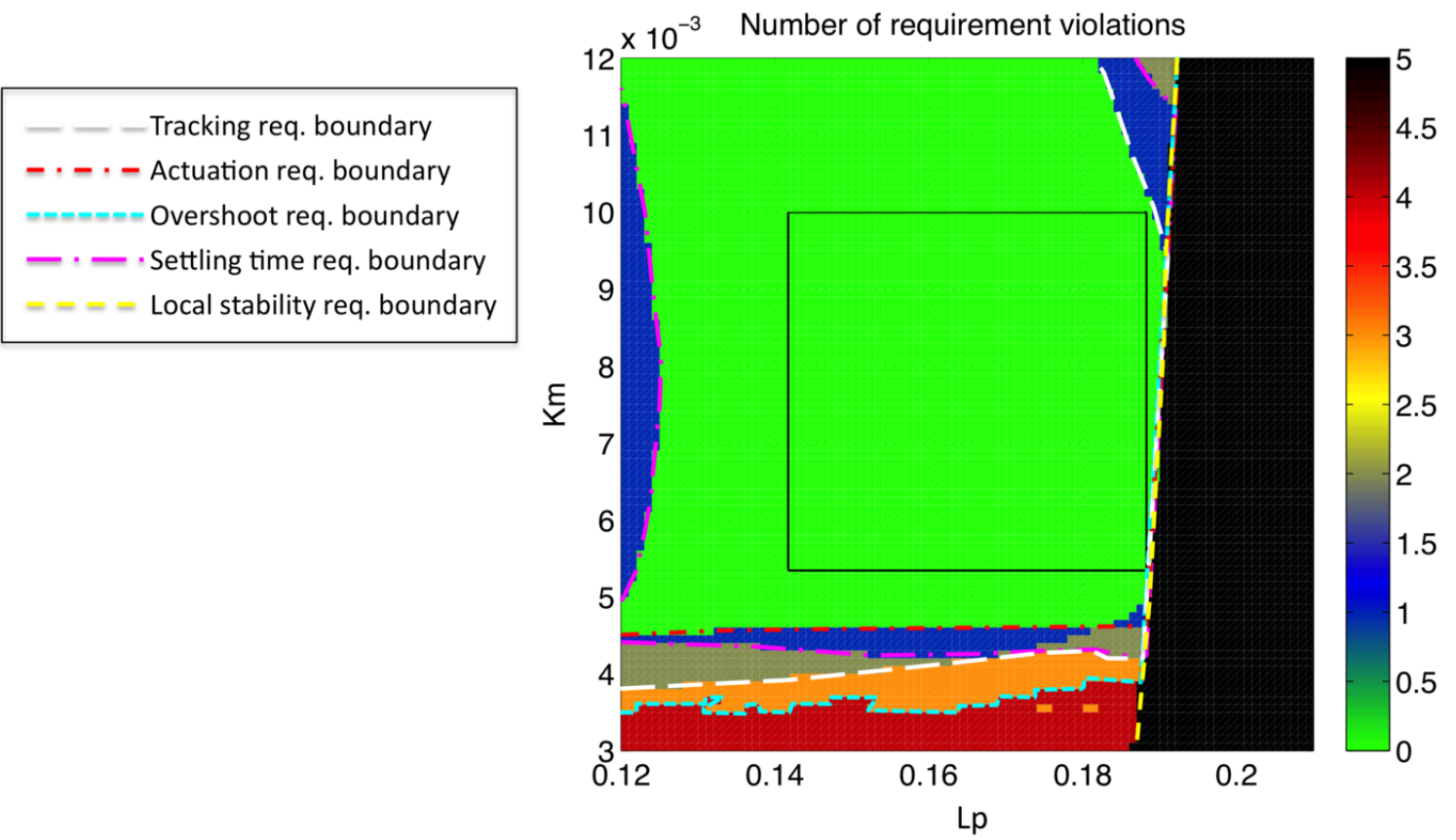

Fig. 10 Number of requirement violations in the parameter space and maximal set for $K_{\text {tuned-2 }}$ according to the nonlinear simulation 


$$
\begin{aligned}
& --- \text { Tracking req. boundary } \\
& -\cdot-\cdot-\text { Actuation req. boundary } \\
& =-=-=\text { Overshoot req. boundary } \\
& -\cdot-\text { Settling time req. boundary } \\
& =--- \text { Local stability req. boundary }
\end{aligned}
$$

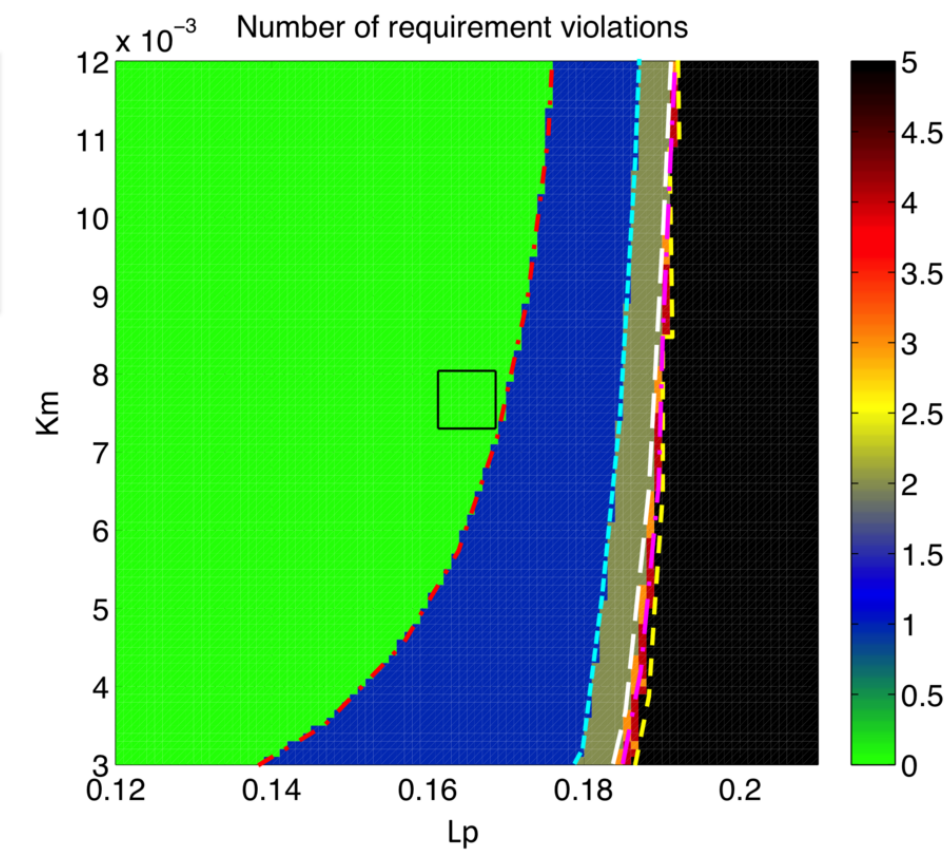

Fig. 11 Number of requirement violations in the parameter space and maximal set for $K_{\text {tuned-2 }}$ according to the linear simulation

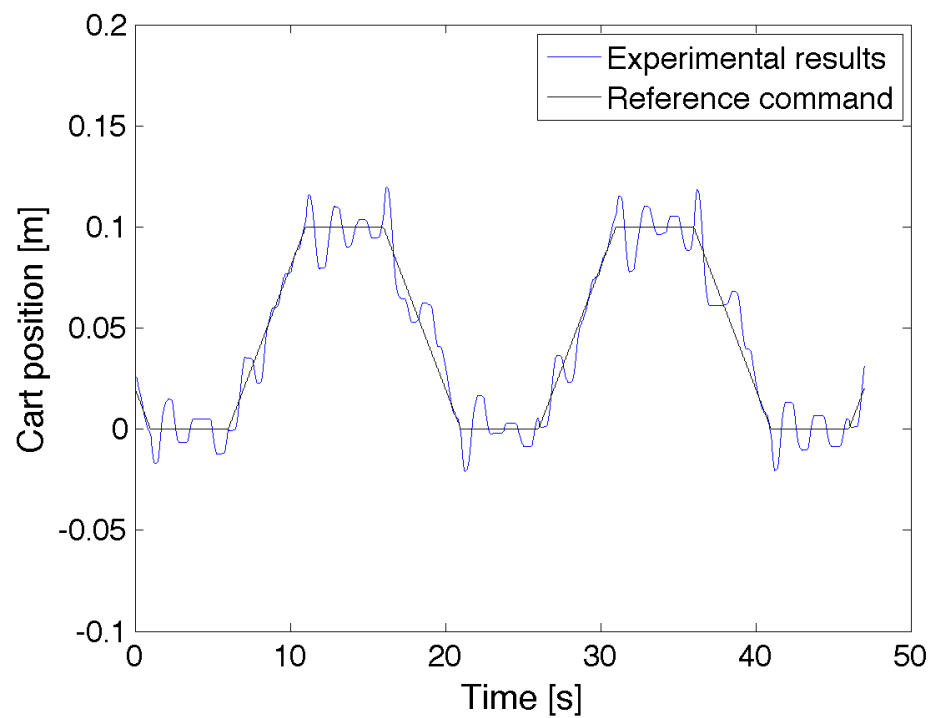

Fig. 12 Experimental time responses for controller $K_{\text {tuned-2 }}$ at $p=\left[0.165,7.67 \times 10^{-3}\right]$ 


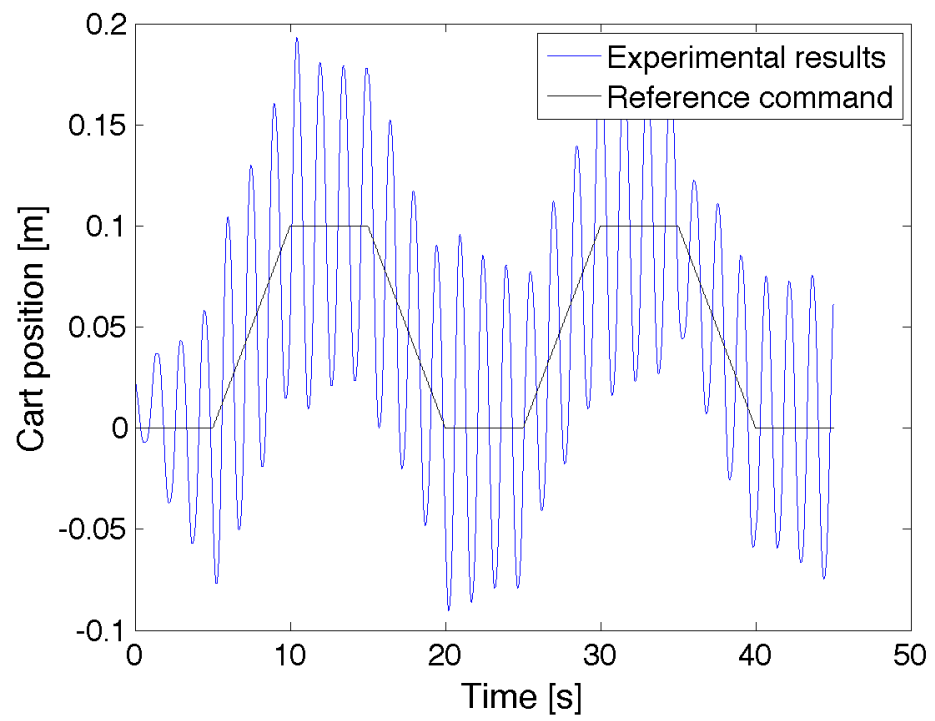

Fig. 13 Experimental time responses for controller $K_{\text {tuned-2 }}$ at $p=\left[0.20,7.67 \times 10^{-3}\right]$

\subsection{Probabilistic Analysis}

This section assesses the probabilistic performance of all of the control designs previously presented. The unified framework in [1] provides explicit methods on how to use maximal sets to compute bounds to failure probabilities. To proceed with failure probability computations, one must first prescribe a PDF for the uncertain parameters. Independent generalized beta distributions were chosen to represent the uncertainty in both $L_{p}$ and $K_{m}$. Generalized beta distributions are parameterized by four values, $[a, b, x \min , x \max ]$, two shaping parameters, a lower limit, and an upper limit, respectively. The specific parameters used in this paper were for $L_{p}$ : [5,5,0.12,0.21], and for $K_{m}$ : $[5,5,0.003,0.012]$. Plots of the probability density functions are given in figure 14 The probabilistic assessments performed in this work were based upon probability bounds computed using maximal sets, and estimates of failure probability using sampling. A robustness comparison of the three controllers is given in table 1 . There are several things to note from these results. In terms of robustness, the baseline controller, $K_{\text {base }}$, shows good robustness when evaluated on the nonlinear system, but very poor robustness when evaluated on the linear system. As stated earlier, this is consistent with the design practice used to generate $K_{\text {base }}$. The controller $K_{\text {tuned }-1}$ had good robustness when evaluated using the linear design model, but suffered greatly when deployed on the nonlinear model. The best choice of controller is $K_{\text {tuned }-2}$ because it has good robustness margins, as indicated by the 46.3 percent improvement in the PSM over $K_{\text {base }}$. These results reinforce two important principles. The first is that parametric uncertainty must be explicitly considered to achieve good robustness, and the second is that model nonlinearities can have a dramatic impact on system robustness. These results clearly demonstrate that controllers designed using linear models may have very poor robustness when evaluated on nonlinear models.

Most of the prior state-of-the-art techniques (e.g., $\mu$-synthesis, $H_{\infty}$, linear parameter varying, etc.) for the analysis and control design in the presence of parametric uncertainty require linear plants and a particular structure of parameter dependencies (affine dependencies, or limited polynomial dependencies). Furthermore, explicitly incorporating time domain specifications into conventional robust control design techniques can be very cumbersome. In contrast, the strategies proposed in this work do not require linear system dynamics or a specific functional form of the uncertainty and can easily accommodate a variety of time or frequency domain requirements. However, one of the greatest advantages is the ease at which the methods used here interface with arbitrary simulation models and the ability to incorporate probabilistic definitions of parametric uncertainty models and therefore represents an advancement in the state of practice for control analysis and design. 

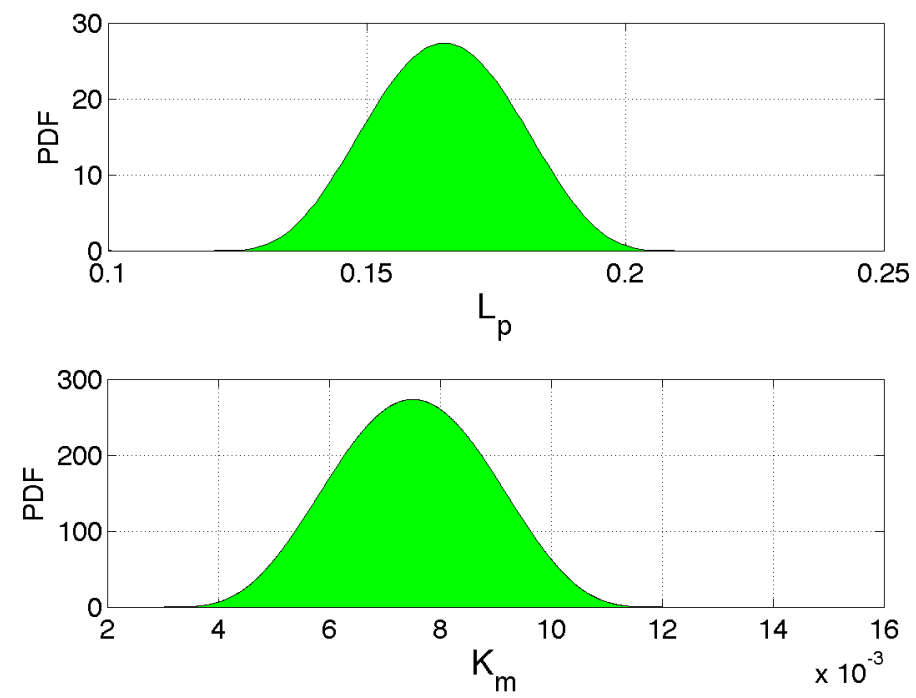

Fig. 14 Probability density functions

\begin{tabular}{|l|l|l|c|c|}
\hline Controller & Test Model & PSM & $\begin{array}{c}\text { Failure Probability } \\
\text { Upper Bound }\end{array}$ & $\begin{array}{c}\text { Failure Probability } \\
\text { Using Sampling }\end{array}$ \\
\hline$K_{\text {base }}$ & Linear & $6.4 \times 10^{-3}$ & 0.8847 & 0.3146 \\
\hline$K_{\text {base }}$ & Nonlinear & $1.6 \times 10^{-2}$ & 0.4538 & 0.1236 \\
\hline$K_{\text {tuned-1 }}$ & Linear & $3.9 \times 10^{-2}$ & $5.22 \times 10^{-4}$ & $1.5455 \times 10^{-7}$ \\
\hline$K_{\text {tuned-1 }}$ & Nonlinear & $6.0 \times 10^{-3}$ & 0.8989 & 0.5035 \\
\hline$K_{\text {tuned-2 }}$ & Linear & $3.7 \times 10^{-3}$ & 0.9604 & 0.3990 \\
\hline$K_{\text {tuned-2 }}$ & Nonlinear & $2.34 \times 10^{-2}$ & 0.1653 & 0.0441 \\
\hline
\end{tabular}

Table 1 Robustness Assessment Results

\section{Conclusions}

This paper presents a control design and analysis methodology for arbitrary dynamic systems in the presence of parametric uncertainty. A brief overview of techniques to compute maximal multidimensional bounding sets and associated probability of failure bounds for parametric uncertainties was given. The classic inverted pendulum was used to demonstrate these techniques for control system design and analysis on a realistic nonlinear system. Generic requirements in the form of command tracking, control actuation, peak overshoot, settling time, and local stability were used to demonstrate the versatility of the method. One of the key results from this work was that the methods are equally applicable to either linear or nonlinear system models, and therefore represent an advancement in the state of practice for control system design and analysis. This technique allows one to use the nonlinear model explicitly in the design process, something many conventional robust control analysis and design methods simply cannot accommodate. Using the nonlinear model in the design process was shown to result in a controller with better robustness properties when applied to the nonlinear system than a controller based on the linear model. 


\section{References}

[1] Crespo, L. G., Kenny, S. P., and Giesy, D. P., "A Computational Framework to Control Verification and Robustness Analysis," NASA TP 2010-216189, NASA Langley Research Center, Hampton, VA, 2010, pp. 1-38.

[2] Franklin, G. F., Powell, J. D., and Emami-Naeini, A., Feedback Control of Dynamic Systems, Prentice Hall, Upper Saddle River, NJ, 6th ed., 2010, pages 37-38.

[3] Crespo, L. G., Munoz, C. A., Narkawicz, A., Kenny, S. P., and Giesy, D., "Uncertainty Analysis via Failure Domain Characterization: Polynomial Requirement Functions," ESREL 2011, Troyes, France, September 2011.

[4] Crespo, L. G., Matsutani, M., and Annaswamy, A., "Verification and Tuning of an Adaptive Controller for an Unmaned Air Vehicle," AIAA Guidance Navigation and Control Conference, Toronto, Canada, August 2010, AIAA-2010-8403. 